TEMAS DE ACTUALIDAD Rev Chil Salud Pública 2014; Vol 18 (2): 192-198

\section{ANÁLISIS RETROSPECTIVO DE LA SITUACIÓN DE VACUNACIÓN ANTIRRÁBICA CANINA EN CHILE ENTRE LOS AÑOS 2002 Y 2012}

\author{
RetrosPeCtive ANALYSIS OF THE STATE OF ANTI-RABIES \\ vaccination in dogs IN ChILE, 2002-2012
}

\title{
RESUMEN
}

El último caso de rabia que afectó a un joven de Quilpué, región de Valparaíso, ha generado nuevamente interés por los perros en las calles. Esto debido a los problemas que ocasionan en salud pública, como por ejemplo mordeduras, y la transmisión de zoonosis, como es el caso de la rabia. La vacunación de los perros contra la rabia es esencial para limitar la transmisión del virus.

Objetivo. La siguiente revisión bibliográfica se centra en estimar la proporción de vacunación antirrábica canina en diferentes ciudades de Chile, entre los años 2002 y 2012.

Metodología. Se utilizó como fuente de información solo memorias de titulo de alumnos de pregrado de tres universidades de Chile, relacionadas con caracterizaciones demográficas $y / o$ aspectos sanitarios de poblaciones caninas.

Resultados. Se observó un promedio de $51,4 \%$ de perros vacunados contra la rabia en estudios en que no se solicitó certificado antirrábico y un $25,1 \%$ de perros vacunados al solicitar certificado.

Discusión. Analizando los porcentajes de vacunación antirrábica en perros en algunas zonas de Chile, es posible establecer que la cobertura de vacunación en estas zonas es inferior al $70 \%$ propuesto por la OIE para poblaciones caninas, lo que conlleva un riesgo en la presentación de casos de rabia en animales y bumanos.

Conclusión. En algunas zonas de Chile hay niveles bajos de vacunación antirrábica en perros, y en ocasiones nulo. Es necesario fomentar la tenencia responsable de animales de compañia y educar acerca de la obligatoriedad anual de la vacuna antirrábica en perros.

Palabras clave: rabia, vacuna, perros, Chile. 


\section{ABSTRACT}

The last rabies case that hit a young man at Quilpue, Valparaiso region, has generated interest again for dogs that can be found in streets. This is due to the problems arising in public health, such as dog bites, and transmission of zoonoses, such as rabies. Vaccination against rabies in dogs is essential to maintain limits to the virus transmission.

Objective. This review focuses on estimating the proportion of canine rabies vaccination in different cities of Chile between 2002 and 2012.

Methodology. Only undergraduate student's theses of three Chilean universities were used as a source of information, all related with demographic characterization and/or sanitary aspects of canine populations.

Results. An average of $51,4 \%$ of dogs vaccinated against rabies was observed in studies that did not request rabies certificate and $25,1 \%$ of vaccinated dogs in studies where rabies certificate was requested.

Discussion. Analyzing the percentages of rabies vaccination in dogs in some places of Chile, it is possible to establish that vaccination coverage in these areas is less than the $70 \%$ proposed by the OIE for dogs populations, which implies a risk of presenting rabies cases in animals and bumans.

Conclusion. In some areas of Chile there are low levels of rabies vaccination in dogs, sometimes being null. Is necessary to promote responsible ownership of companion animals and educate about the mandatory annual rabies vaccine in dogs.

Key words: rabies, vaccine, dogs, Chile.

\section{INTRODUCCIÓN}

La rabia (hidrofobia) es una enfermedad viral aguda, zoonótica y de carácter letal para la mayoría de las especies. Puede presentarse en mamíferos domésticos como silvestres. La transmisión se produce principalmente por mordeduras, a través de la saliva del animal infectado. En el hombre la letalidad es cercana al $100 \%$, con casos excepcionales de personas que han sobrevivido al virus. ${ }^{1}$ Existe vacuna para la enfermedad, tanto para animales domésticos, ${ }^{2}$ así como también vacunación preexposición al virus para humanos en riesgo de contagio y posexposición en casos sospechosos. ${ }^{3}$

Para el ciclo urbano de la rabia, el perro es el principal mamífero que puede transmitir el virus hacia el ser humano, ${ }^{4}$ especialmente los perros callejeros, debido a la cercanía e intimidad de esta especie con el hombre, y por ser estos los de mayor porcentaje en las calles. Es importante mencionar la diferencia que existe entre perro vagabundo y perro callejero. El perro vagabundo es aquel que no tiene dueño ni tampoco una vivienda asociada, mientras que el perro callejero es aquel que tiene dueño y vivienda asociada, pero que las personas dejan salir y pasan la mayor parte del tiempo en la calle. ${ }^{5}$

En el año 2010, Chile fue declarado como país libre de rabia canina (variante canina 1 y 2) ante la OMS y OIE. ${ }^{6}$ Sin embargo, el virus de la rabia sigue presente en el país en murciélagos insectívoros (ciclo silvestre), principalmente Tadarida brasiliensis, especie a la cual se asocia la variante 4 del virus. ${ }^{7}$ Esto significa un riesgo de contagio para mamíferos domésticos y humanos, por lo que es relevante vacunar a los animales de compañía contra la rabia. ${ }^{8}$

En agosto del año 2013, el ISP confirmó un nuevo caso de rabia humana en un joven de Quilpué, región de Valparaíso, con el antecedente de haber sido mordido por un perro callejero. ${ }^{9}$ Es el primer caso de rabia humana en Chile tras la mordida de un perro desde 1972. ${ }^{8}$ En aquella ocasión se pudo detectar 
que la variante del virus era canina, sin embargo en el caso de Quilpué se señala una cadena de transmisión murciélago/perro/ persona. ${ }^{10}$

Ante lo acontecido, ha tomado relevancia nuevamente el tema de los perros vagabundos y callejeros, que hace años en Chile constituyen un problema de salud pública, sin existir una legislación adecuada. Si bien existe una baja ocurrencia de transmisión de rabia en el país, la probabilidad de que un perro enfermo de rabia infecte a una persona se ve aumentada debido al creciente número de perros en las calles cada año, como lo observado en algunas ciudades de Chile, ${ }^{11} \mathrm{y}$ los eventos de mordeduras asociados a ellos. ${ }^{12,13}$

La presente revisión tiene por objetivo estimar la proporción de vacunación antirrábica en caninos, en algunas comunas y localidades de Chile, a partir del año 2002, debido a lo preocupante que resulta que el último caso de rabia humana sea por mordedura de perro.

\section{MATERIALES Y MÉTODO}

Para recopilar información, se utilizó una fuente de datos secundaria, mediante revisión de un total de 24 memorias de título de pregrado de alumnos de Medicina Veterinaria, correspondientes a la Universidad de Chile (UCh), Universidad de Concepción (UdeC) y Universidad Austral de Chile (UACh). La revisión de las memorias de título fue realizada por una sola persona, y se tuvo acceso a los textos a través de las bibliotecas de cada facultad o vía electrónica por sus bibliotecas en línea.

El criterio de búsqueda fue que las memorias correspondieran a caracterizaciones demográficas y/o aspectos sanitarios de poblaciones caninas en diferentes comunas o localidades de Chile, en las cuales se realizó una estimación de la proporción de perros vacunados contra la rabia en aquel lugar. Las memorias seleccionadas recolectaron los datos entre el año 2002 y 2012, a través de encuestas.

La variable a analizar fue el número de peros vacunados del total de caninos en cada ciudad o localidad estudiada, para establecer la proporción de vacunación antirrábica. También se analizó si el propietario contaba con el certificado antirrábico canino al momento de realizar la encuesta, considerándose vacunación vigente al presentarlo, mientras que si no lo tenía, se consideró al perro sin vacunación vigente. En las memorias en que no se solicitó certificado antirrábico al momento de la encuesta, solo se consideró la respuesta del propietario.

Se ingresaron los resultados a una base de datos en Microsoft Excel $^{\circledR}$, y luego se utilizó estadística descriptiva mediante frecuencias relativas y medianas del total de perros vacunados por año. Se evaluó la significancia de la diferencia entre dos proporciones independientes (prueba de z) utilizando la herramienta Vassarstats. ${ }^{14}$ Para este análisis solo se utilizó el porcentaje global de los años 2002, 2004 y 2009, dado que en estos años se aplicaron encuestas en las que no se pidió certificado antirrábico y también encuestas en las que sí se solicitóel documento.

\section{RESULTADOS}

De las 24 memorias de título analizadas, 5 corresponden a las UCh, 9 a la UdeC y 10 a la $\mathrm{UACh}$, que corresponden a estudios en ocho regiones del país.

En la Tabla 1 se observa los porcentajes de perros vacunados de cada comuna o localidad, con y sin certificado antirrábico, por orden cronológico respecto a la recolección de los datos desde el año 2002 al 2012. Se incluye además autor, lugar del estudio y universidad.

En la Figura 1 se aprecia que las medianas de vacunación antirrábica por año no alcanzan un $70 \%$. Se puede observar que la tendencia, sin hacer diferencia entre con y sin certificado, es que las vacunaciones van en aumento hasta el año 2009, y luego hay una tendencia a la baja al exigir certificado. El promedio de perros vacunados en estudios donde se solicitó certificado fue de un $25,1 \%$, mientras que el promedio en aquellos estudios en donde no se solicitó certificado antirrábico fue de un $51,4 \%$. Al analizar las proporciones de vacu- 
nación con y sin certificado antirrábico de los años 2002, 2004 y 2009, se observan diferencias estadísticamente significativas.

\section{DISCUSIÓN}

En el estudio realizado por Guajardo (2003) en Santa Cruz, es preocupante el bajo porcentaje de vacunación ya que esta ciudad es cercana al lugar del último caso de rabia humana antes del caso del 2013, el cual se produjo en 1996 (ver Tabla 1). ${ }^{15}$ Aunque en ese caso el contagio fue por contacto con murciélago, se debe tener en cuenta ya que estos eventualmente podrían transmitir el virus a animales domésticos como el perro.

Cabe destacar que en los estudios realizados en las ciudades de Panguipulli16 y La Unión ${ }^{17}$ en el 2003, el porcentaje obtenido se vio influido por la realización previa de campañas de vacunación masiva por parte del Servicio de Salud. Lo mismo sucedió en el estudio de Zúñiga (2005) en Valdivia, pero en este caso debido a que la autoridad sanitaria realizó vacunaciones masivas en perros durante los años 2004 y 2005, por la alta prevalencia histórica de casos positivos de rabia en murciélagos de la ciudad de Valdivia. ${ }^{18}$ Algo similar pasó en el estudio de Boettcher (2012) en Niebla, ya que ese año la Municipalidad, en conjunto con la Facultad de Medicina Veterinaria de la UACh, realizó vacunaciones masivas de perros antes de realizar la encuesta (ver Tabla 1). ${ }^{19}$

Se puede apreciar que en los estudios donde se exigió el certificado antirrábico el porcentaje de vacunación alcanzó un 49,3\%, el cual está por debajo de lo deseable por la OIE, que establece que al menos un $70 \%$ de los perros debe tener vacunación antirrábica vigente para controlar la rabia en una zona determinada. ${ }^{20}$ Esto difiere de aquellos estudios donde no se solicitó el certificado antirrábico, puesto que los porcentajes de vacunación en ellos alcanzan hasta un $74,9 \%$ de cobertura, lo que se acerca a lo recomendado por la OIE. Cabe destacar que en estos casos solo se confiaba en la respuesta del propietario, por lo que puede darse sesgos en la información recopilada, dado que no se puede saber si el perro tenía su vacunación antirrábica vigente o si solo fue vacunado alguna vez en su vida. Si bien la presente revisión bibliográfica tiene un acotado número de memorias de título analizadas y regiones del país que no se ven representadas en la muestra, se debe tener en consideración que en Chile existe el Reglamento de prevención de la rabia en el hombre y los animales, en el cual está establecida la obligatoriedad para los propietarios de someter a sus perros anualmente a la vacunación antirrábica, ${ }^{2}$ condición que según los resultados no se ha cumplido en algunas zonas del país en los últimos años.

Considerando la baja vacunación antirrábica en caninos en algunas ciudades de Chile, la presencia de la variante 4 de rabia transmitida por murciélagos, y antecedentes de no uso de medio de sujeción y no mantención en confinamiento de los perros, ${ }^{19,21,22}$ entre otros, indican que existe un riesgo para la salud pública de presentación de casos de rabia, ${ }^{8}$ como lo ocurrido en Quilpué, pese a que Chile es considerado país libre de rabia canina por organismos internacionales. ${ }^{6}$ Es interesante tener en cuenta que la década analizada con estos estudios es marcada por este hito, puesto que se observó en general un aumento del porcentaje de vacunación hasta el año 2009, un año antes de que Chile fuera declarado libre, luego de lo cual hay una disminución en este porcentaje. La información contenida en las memorias de título evaluadas permitió un análisis descriptivo en conjunto para aportar información relacionada con la rabia en nuestro país.

\section{CONCLUSIÓN}

Se pudo determinar que en la década 20022012 hubo un bajo porcentaje de perros vacunados en algunas localidades de Chile $(0 \%),{ }^{23,24,25}$ mientras en otros lugares se pudo observar que este porcentaje superaba el $50 \%{ }^{26,34,35,37,38}$ sin presentar certificado antirrábico.

Es de suma importancia fomentar la educación sanitaria respecto a los animales de 
compañía, tenencia responsable y zoonosis, como también facilitar o ampliar la entrega de información a los propietarios acerca de sus obligaciones, como por ejemplo mantener a los animales con su vacunación antirrábica al día. Por otro lado, es necesario mejorar e implementar medidas a nivel país, como programas de vacunación masiva de perros y control de poblaciones caninas, sumado a una legislación adecuada capaz de regular las acciones para este fin.

\section{REFERENCIAS}

1. México. Secretaría de Salud. Subsecretaría de Prevención y Promoción de la Salud. Dirección General de Epidemiología. Manual de procedimientos estandarizados para la vigilancia epidemiológica de la rabia en bumano. México: Secretaría de Salud. Subsecretaría de Prevención y Promoción de la Salud. Dirección General de Epidemiología; 2012.

2. Reglamento de prevención de la rabia en el hombre y en los animales. Dto. núm. 89/02. Publicado en el Diario Oficial (8.01.2003).

3. World Health Organization. Rabies vaccines: WHO position paper-Recommendations. Vaccine. 2010; 28: 7140-7142.

4. Chile. Ministerio de Salud. Manual de procedimientos de medidas ambientales para el control y prevención de rabia animal. Santiago: MINSAL; 2008.

5. Ibarra L, Espínola F, Echeverría M. Una prospección a la población de perros existente en las calles de la ciudad de Santiago, Chile. Av Cs. Vet. 2006; 21: 33-39.

6. Chile. Ministerio de Salud. Departamento de epidemiología. Vigilancia de la rabia en Chile [en línea]; 2013 [consultado 12.01.2014]. Disponible en: http://epi.minsal. cl/epi/html/bolets/reportes/rabia/Vigilancia_ Rabia_act.pdf

7. Favi M, De Mattos C, Yung V, Chala E, López L.. First case of human rabies in Chile caused by an insectivorous bat virus variant. Emerg Infect. Dis. 2002 Jan; 8(1): 79-81.

8. Favi M, Pavletic C. La rabia animal en Chile. El Vigía. 2002; 6(17): 15.
9. Chile. Ministerio de Salud. Reporte caso de rabia humana. Valparaíso: MINSAL; 2013.

10. Chile. Ministerio de Salud [en línea]. Santiago; c2013. [actualizado 25.11.2013; consultado 20.04.2014]. [aprox. 2 pantallas]. Disponible en: http://web.minsal.cl/node/376

11. Rossi P. Descripción demográfica y de algunos indicadores de tenencia responsable de la población canina y felina en la ciudad de Chillán año 2005 [tesis]. Chillán: Universidad de Concepción; 2006.

12. Universidad de Chile. Facultad de Ciencias Veterinarias y Pecuarias [en línea]. Santiago: Comunicaciones Favet; c2013. [actualizado 12.08.2013; consultado 13.01.2014]. [aprox. 3 pantallas]. Disponible en: http:// www.uchile.cl/noticias/93843/caso-de-rabiaen-chile

13. Ibarra L, Morales MA, Cáceres L. Mordeduras a personas por ataques de perros en la ciudad de Santiago, Chile. Av. Cs. Vet. 2003; $18(1$ y 2$): 41-46$

14. VassarStats [en línea]. New York: Richard Lowry; c2014. [consultado 14.01.2014]. Disponible en: http://vassarstats.net/

15. Guajardo JA. Caracterización de las poblaciones caninas y felinas de la ciudad de Santa Cruz, VI región, Chile [tesis]. Chillán: Universidad de Concepción; 2003.

16. Valenzuela JP. Estudio de algunas características demográficas de la población canina de Panguipulli y determinación del grado de consultas hospitalarias por mordeduras [tesis]. Valdivia: Universidad Austral de Chile; 2003.

17. Gallardo M. Características demográficas de la población canina y recuento de la población felina en la ciudad de La Unión [tesis]. Valdivia: Universidad Austral de Chile; 2003.

18. Zúñiga ML. Características demográficas de la población canina y recuento de la población felina, en la ciudad de Valdivia, Chile [tesis]. Valdivia: Universidad Austral de Chile; 2007.

19. Boettcher K. Caracterización de la población canina y nivel de conocimiento de distemper de sus propietarios en la localidad de Niebla, provincia de Valdivia [tesis]. Valdivia: Universidad Austral de Chile; 2012. 
20. World Health Organization. National programmes for the control of rabies in dogs [en línea]. Ginebra: WHO Experts Consultation on Rabies; 2005. [consultado 17.01.2014]. Disponible en: http://www.who. int/rabies/animal/ExcerptTRS931.pdf?ua=1

21. San Martín C. Desarrollo de un indicador de tenencia responsable en base a la caracterización demográfica de la población canina en Talcahuano en el año 2009 [tesis]. Concepción: Universidad de Concepción; 2010.

22. Ovalle R. Caracterización demográfica de la población canina y conocimiento sobre zoonosis en la población de la localidad de Dichato, región del Biobío, Chile [tesis]. Concepción: Universidad de Concepción; 2013.

23. Nadeau A. Caracterización de la población canina y felina en la ciudad de Bulnes [tesis]. Chillán: Universidad de Concepción; 2003.

24. Andrade R. Características demográficas de la población canina y recuento de felinos en la ciudad de Los Muermos, décima, región, Chile [tesis]. Valdivia: Universidad Austral de Chile; 2003.

25. Riquelme M. Caracterización de la población canina y felina de la ciudad de Quillón, VIII región, Chile [tesis]. Chillán: Universidad de Concepción; 2004.

26. Bustamante S. Demografía en las poblaciones de perros y gatos en la comuna de Santiago. Santiago: Universidad de Chile; 2008.

27. Alvarado S. Caracterización de las poblaciones caninas y felinas en la ciudad de San Carlos, octava región [tesis]. Chillán: Universidad de Concepción; 2002.

28. Carnevali R. Estudio de algunas características demográficas, de manejo de la población canina y grado de consulta hospitalaria por mordeduras de perros durante el año 2002 en el pueblo de Alerce, comuna de Puerto Montt, décima región [tesis]. Valdivia: Universidad Austral de Chile; 2003.
29. Werlinger F. Demografía en las poblaciones de perros y gatos en la comuna de La Pintana [tesis]. Santiago: Universidad de Chile; 2003.

30. Rojas C. Caracterización de la población canina y felina en la ciudad de Antofagasta [tesis]. Chillán: Universidad de Concepción; 2005.

31. Torres H. Estudio de características demográficas de la población canina en la ciudad de Lanco y nivel de conocimiento de sus propietarios sobre alguna zoonosis [tesis]. Valdivia: Universidad Austral de Chile; 2003.

32. Bravo Y. Censo de la población canina y estimación del grado de consulta hospitalaria por mordeduras en la localidad de Chacao, provincia de Chiloé [tesis]. Valdivia: Universidad Austral de Chile; 2003.

33. Catrilef M. Censo de la población canina y estimación del grado de consulta hospitalaria por mordeduras en la ciudad de Frutillar [tesis]. Valdivia: Universidad Austral de Chile; 2004.

34. Rojas A. Demografía en las poblaciones de perros y gatos en la comuna de Lo Prado [tesis]. Santiago: Universidad de Chile; 2005.

35. Illanes J. Demografía en las poblaciones de perros y gatos en el área rural y urbana de la comuna de Calera de Tango [tesis]. Santiago: Universidad de Chile; 2009.

36. Araus D. Características demográficas, sanitarias, de manejo y mordeduras denunciadas de la población canina, durante el período 2008-2009, en la ciudad de Puerto Aysén, Chile [tesis]. Valdivia: Universidad Austral de Chile; 2009.

37. Recabarren C. Algunos aspectos sanitarios de la población de perros y gatos en la comuna de Viña del Mar, región de Valparaíso [tesis]. Santiago: Universidad de Chile; 2013.

38. Jorquera MF. Caracterización demográfica y condiciones de tenencia de la población canina en la zona centro de la ciudad de Chillán, Chile [tesis]. Chillán: Universidad de Concepción; 2011. 


\section{ANEXOS}

Tabla 1. Porcentajes de perros vacunados, con y sin certificado de vacunación antirrábica, en ciudades de Chile, por orden cronológico de acuerdo al año de recolección de los datos $(n=24)$.

\begin{tabular}{|c|c|c|c|c|c|}
\hline \multirow{2}{*}{$\begin{array}{c}\text { Año } \\
\text { datos }\end{array}$} & \multirow[t]{2}{*}{ Autor } & \multirow[t]{2}{*}{ Ciudad (Región) } & \multirow[t]{2}{*}{ Univ. } & \multicolumn{2}{|c|}{ Perros con vacunación antirrábica } \\
\hline & & & & $\%$ con cert. & $\%$ sin cert. \\
\hline \multirow[t]{5}{*}{2002} & Nadeau (23) & Bulnes (Biobío) & UdeC & 0 & - \\
\hline & Alvarado (27) & San Carlos (Biobío) & UdeC & 6 & - \\
\hline & Carnevali (28) & Alerce (Los Lagos) & UACh & 8,2 & - \\
\hline & Andrade (24) & Los Muermos (Los Lagos) & UACh & 0 & - \\
\hline & Werlinger (29) & La Pintana (Metrop.)* & $\mathrm{UCh}$ & - & 27,5 \\
\hline \multirow[t]{6}{*}{2003} & Rojas (30) & Antofagasta (Antofagasta) & UdeC & 8,9 & - \\
\hline & Guajardo (15) & Santa Cruz (O’Higgins) & UdeC & 18,4 & - \\
\hline & Valenzuela (16) & Panguipulli (Los Ríos) & UACh & 49,3 & - \\
\hline & Gallardo (17) & La Unión (Los Ríos) & UACh & 46,6 & - \\
\hline & Torres (31) & Lanco (Los Ríos) & UACh & 22,7 & - \\
\hline & Bravo (32) & Chacao (Los Lagos) & UACh & 7 & - \\
\hline \multirow[t]{3}{*}{2004} & Riquelme (25) & Quillón (Biobío) & UdeC & 0 & - \\
\hline & Catrilef (33) & Frutillar (Los Lagos) & UACh & 13,4 & - \\
\hline & Rojas (34) & Lo Prado (Metrop.)* & $\mathrm{UCh}$ & - & 66,3 \\
\hline \multirow[t]{2}{*}{2005} & Rossi (11) & Chillán (Biobío) & UdeC & 43,6 & - \\
\hline & Zuñiga (18) & Valdivia (Los Ríos) & UACh & 43,7 & - \\
\hline \multirow[t]{2}{*}{2007} & Illanes (35) & Calera de Tango (Metrop.)* & UCh & - & 58,5 \\
\hline & Bustamante (26) & Santiago (Metrop.)* & $\mathrm{UCh}$ & - & 74,9 \\
\hline \multirow[t]{3}{*}{2009} & Araus (36) & Puerto Aysén (Aysén) & $\mathrm{UACh}$ & - & 21 \\
\hline & Recabarren (37) & Viña del Mar (Valparaiso) & UCh & - & 54,7 \\
\hline & San Martín (21) & Talcahuano (Biobío) & UdeC & 46,8 & - \\
\hline 2010 & Jorquera (38) & Chillán Centro (Biobío) & $\mathrm{UdeC}$ & - & 58,7 \\
\hline \multirow[t]{2}{*}{2012} & Ovalle (22) & Dichato (Biobío) & UdeC & 24,9 & - \\
\hline & Boettcher (19) & Niebla (Los Ríos) & UACh & 35,3 & - \\
\hline
\end{tabular}

*Metrop.: Metropolitana

Figura 1. Tendencia de las medianas de vacunación antirrábica en perros de algunas ciudades de Chile, 2002 - 2012.

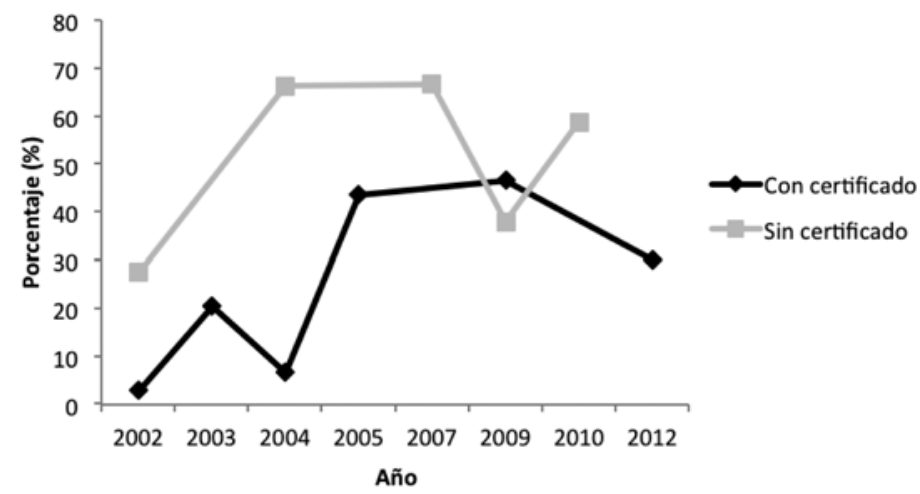

